\title{
WOOD FRACTURE PATTERN DURING THE WATER DESORPTION PROCESS
}

\author{
Liyu Wang ${ }^{1}$, Guangjie Zhao ${ }^{2}$, Zhenyou $\mathrm{Lu}^{3}$
}

\begin{abstract}
Wood is a hygroscopic material and its mechanical properties change with moisture content below the fiber saturation point. Fracture characteristic is one of most important mechanical properties of wood and often is taken as an important safety design factor for wood as an engineered structural material. The aim of this paper is to investigate the effect of moisture content changes on wood fracture properties, focusing on fracture behavior and patterns with different crack orientations of Betula platyphylla Suk. and Pinus bungeana in two different desorption processes. These two desorption processes were, respectively, from fiber saturation point to an equilibrium moisture content corresponding to $82.4 \% \mathrm{RH}$ at $20^{\circ} \mathrm{C}$ and from $82.4 \% \mathrm{RH}$ to $35.2 \% \mathrm{RH}$ at the same temperature of $20^{\circ} \mathrm{C}$.
\end{abstract}

Keywords: water desorption, wood fracture

\section{INTRODUCTION}

Wood fracture performance is influenced by temperature and moisture content with moisture content having significant impact. Smith and Chui (1994) sampled material in green condition from flat sawn boards from three premature red pines grown in New Brunswick, Canada. They concluded that reduction in moisture content at test from the fiber saturation point to $18 \%$ resulted in an increase in mode I energy for complete separation of fracture surfaces and crack growth in the longitudinal direction. However, below $18 \% \mathrm{MC}$, any reduction in $\mathrm{MC}$ at test led to a reduction in fracture energy. Kretschmann and Green (1996) studied effects of moisture content and specific gravity for clear southern pine and concluded that mode II stress intensity factor increases as MC decreases, reaching a maximum between 10 and $12 \% \mathrm{MC}$ for all levels of density. They also found that mode I stress intensity factor increases as MC decreases, reaching a maximum between 7 and 9\% MC for all levels of density. In our previous paper (Wang et al. 2003), wood fracture pattern was investigated under different relative humidity environment in moisture adsorption process. The objective of this work was to investigate the wood fracture behavior in moisture desorption process on the effect of water transition on wood fracture properties, using a similar techniques as discussed in the previous paper (Wang et al. 2003).

\footnotetext{
${ }^{1}$ School of Technology, Beijing Forestry University, Qinghua East Road 35, Haidian District, Beijing, P.R.China, 100083. E-mail: liyuwang2000@yahoo.com.cn

${ }^{2}$ College of Material Science and Technology, Beijing Forestry University, Qinghua East Road 35, Haidian District, Beijing, P.R.China, 100083. Email: zhaogjws@263.net.

${ }^{3}$ School of Technology, Beijing Forestry University, Qinghua East Road 35, Haidian District, Beijing, P.R.China, 100083. E-mail: Luzy@bjfu.edu.cn Corresponding author: : liyuwang2000@yahoo.com.cn

Received: 'October 24, 2005. Accepted: March 26, 2006
} 


\section{EXPERIMENT MATERIALS AND METHODS}

\section{Test specimen preparation}

In the experiment, Betula platyphylla Suk. and Pinus bungeana were used and the sampling method followed the Chinese National Standard GB1927-91(1992):for tree sampling collection for physical and mechanical tests of wood. Specimens included four different orientations TL, TR, LR, LT planes. Here, the first letter denotes direction normal to crack plane and the second one denotes direction of crack. An edge crack was cut in the center of the specimen using a fine saw blade and the crack tip was sharpened. The TL and LT specimen dimensions were 85 (length) x 15 (width) x $5 \mathrm{~mm}$ (thickness). The corresponding dimensions for TR samples were $95 \times 20 \times 10 \mathrm{~mm}$, and those for LT samples (Pinus bungeana) were $120 \times 20 \times 10 \mathrm{~mm}$.

\section{Fracture testing}

The strong and weak degree of stress field near the tip of crack is determined by stress intensity factor $\mathrm{K}_{\mathrm{I}}$. Plane strain fracture toughness $\mathrm{K}_{\mathrm{IC}}$ is the critical stress intensity factor when wood crack starts to propagate under static loading. Specimens were tested by universal mechanical testing machine at a rate of $0.5 \mathrm{~mm} / \mathrm{min}$ and for each specimen, images were captured at various load levels up to failure. The test method of fracture toughness followed the Chinese National Standard GB4161-84 (1985) for plane-strain fracture toughness of metallic materials. This method is not completely suitable for an orthotropic material such as wood, but it is acceptable when the orthotropic main axis direction is superposed with the crack surface direction, as well as the crack propagation direction. Therefore, the above method is suitable for TL and TR specimens because of their crack propagation along the wood initial crack. But for LR, LT specimens, as crack growth sloped from the initial crack direction, the fracture toughness value measured can only be considered nominally for reference.

\section{Water desorption experiment}

In order to inspect wood fracture pattern under different relative humidity in desorption process, the samples were initially dried to the fibre saturation point and then exposed, respectively, to $82.4 \%$ $\mathrm{RH}$ and $35.2 \% \mathrm{RH}$ at $20^{\circ} \mathrm{C}$. The relative humidity environment was created by the sulfuric acid watery solution and the temperature was controlled by a constant temperature box. During the desorption process, some specimens were taken out at different times to determine their moisture content and fracture nature.

\section{RESULT AND DISCUSSION}

\section{Wood fracture pattern in the first desorption step $(82.4 \% \mathrm{RH})$}

\section{Crack propagation path}

In the desorption process, specimens' crack propagation path was the same as that in the moisture absorption process (Wang et al., 2003). Regardless of specimen types, all of their crack propagation occurred at the crack tips. The crack propagation direction of TL and TR specimens was consistent with the original crack direction, and the fracture occurred as soon as their cracks were initiated. However, the crack of LT specimen started along initial crack direction, but, after very short period of time, it changed the direction and propagated along the fiber direction. The crack propagation of LR specimen was complex, starting along initial crack direction in very short period of time and then propagating in folding linear direction as shown in Figure 1.

As observed from both the adsorption in previous work (Wang et al., 2003) and from this work, the crack propagation path has the same pattern and is not by moisture content change. 


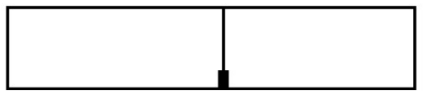

TL

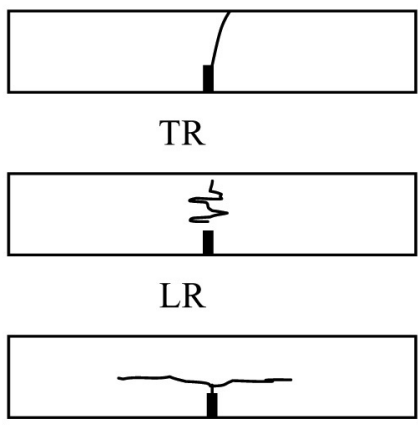

LT

FIGURE 1. Crack propagation pattern in desorption process for the four types of specimens
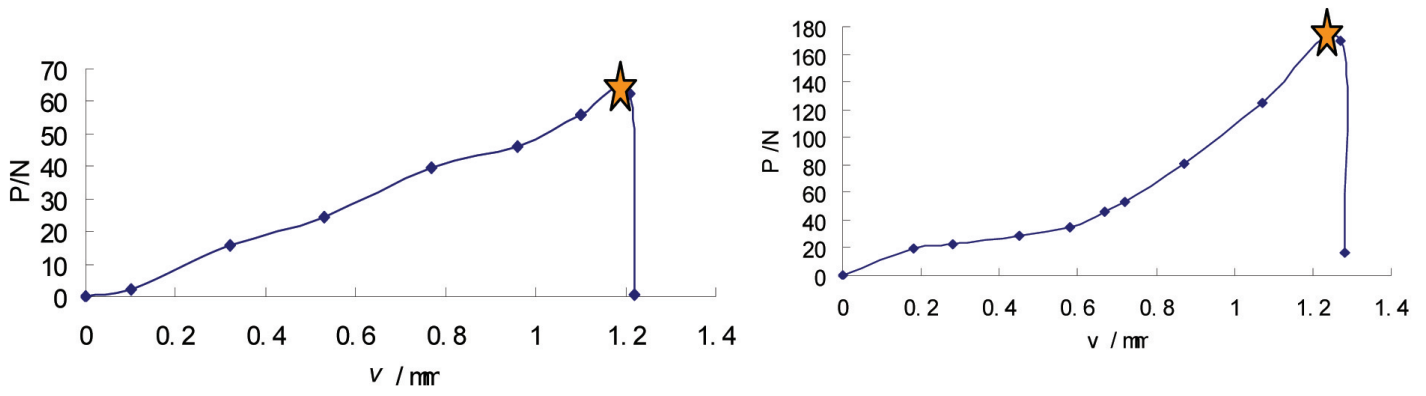

TL

TR

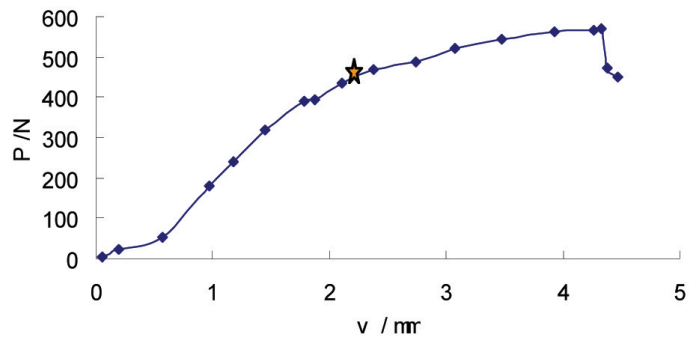

LR

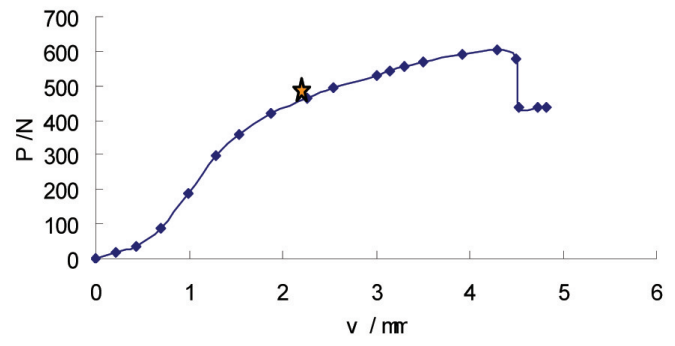

LT(Pinus bungeana)

FIGURE 2. Load and displacement curve for specimens during the first step of desorption to $82,4 \% \mathrm{RH}$.

\section{Critical load determination}

From the experiments for TL and TR specimens, it was observed that the fracture occurred as soon as the crack started to expand. Or in other words crack did not have obvious subcritical expansion. In these cases, the maximum load when the specimen ftacured is termed as the critical load, and the measured loading and displacement curves in the first step of desorption are illustrated in Figure 2.

From Figure 2, it is seen that for TL and TR specimens, the curve of load (P) versus displacement (V) follows an approximate linear relation in the early stage of the loading, and the critical load nearly 
superposed the maximum load. Once crack started to grow, the load rapidly dropped and the test specimens would fracture.

But for LT, LR specimens, crack would propagate slowly with different degrees and no obvious symptoms were observed when crack began to propagate before specimens fracture. From the point, the maximum fracture load was no longer taken as the critical load. During specimen testing, «burst out» sound was frequently heard clearly during rupture. Thus the 1 st «burst out» load was taken as the critical load $P_{\mathrm{Q}}$ as indicated by a start in Figure 2. Seen from figure 2, even if reaching the critical load $P_{\mathrm{Q}}$, at which crack started to lose stability and propagate, the load continues to increase with crack growth. The specimen still has high load-bearing capacity after the 1st rupture. After reaching the largest load Pmax, the load dropped with crack growing and displacement increasing. By this point the specimen had gradually lost its load-bearing capacity.

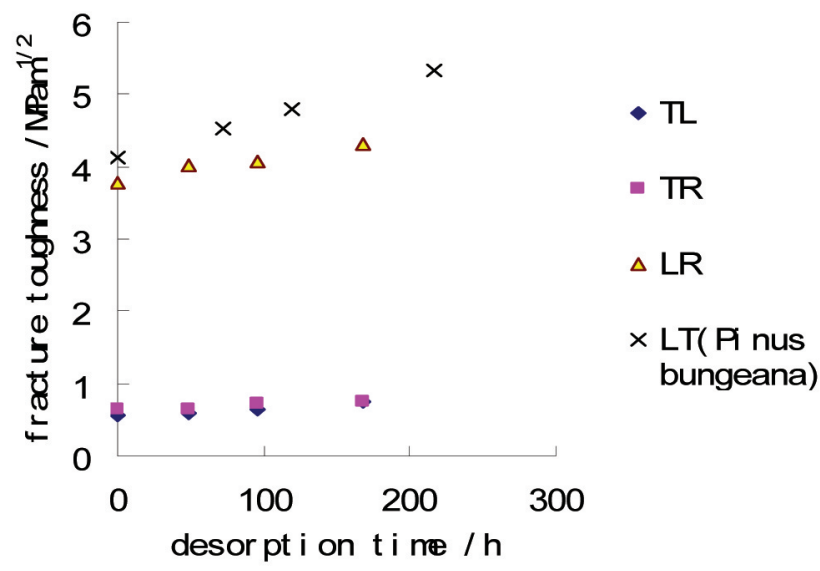

FIGURE 3 Fracture toughness of specimens during the first desorption stage at $20^{\circ} \mathrm{C}(82.4 \% \mathrm{RH})$

\section{Fracture toughness}

Figure 3 shows the results of fracture toughness at different elapsed time during the first stage of desorption $(82.4 \% \mathrm{RH})$. Different specimen types had different fracture toughness values. But for identical specimen types under different time of equilibrium, their fracture toughness values were different. Specimen fracture toughness increased gradually with elapsed time during desorption, and the fracture toughness of TL and TR specimens was quite close and its value was much lower than those of LR and LT specimens.

\section{Wood fracture pattern in the second moisture desorption stage (82.4\% to $35.2 \% \mathrm{RH})$}

\section{Crack propagation path}

In the second desorption process, specimens' crack propagation path was basically the same as that in the first desorption process. Thus the results in Figure 1 can represent those for specimens in the second desorption process.

\section{Critical load determination}

The maximum load is higher in the second stage of desorption as the moisture content is lower. As can be seen from the experiment, the moisture desorption process would not strongly influence the critical load. Thus a similar critical load determination method to the first moisture desorption process may be used by the second moisture desorption process. With desorption time growth in the moisture desorption process, TL, TR specimens would retard a while to fracture after getting up crack. Because the time was very short, only several seconds, therefore the biggest load was thought as the critical load. 
Regarding LT, LR specimens, critical load was still the load at some moment when the first clearly " rupture» sound was heard.

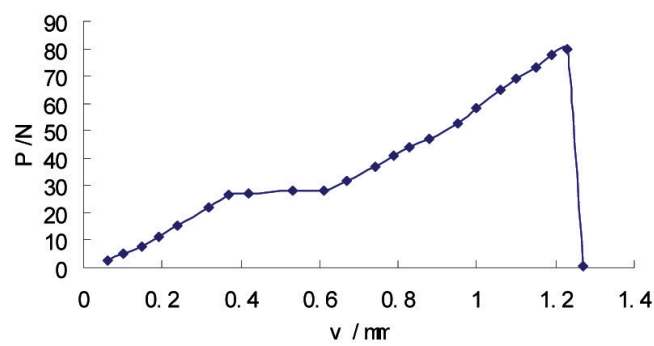

TL

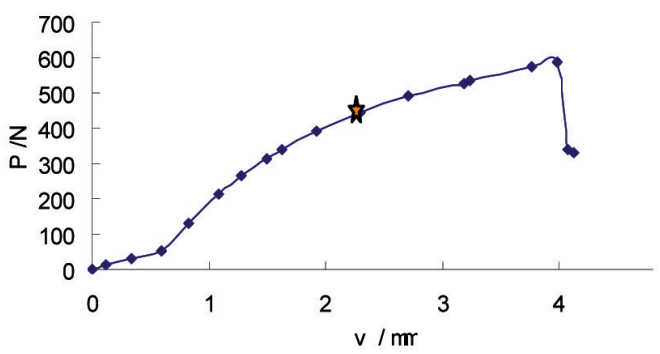

LR

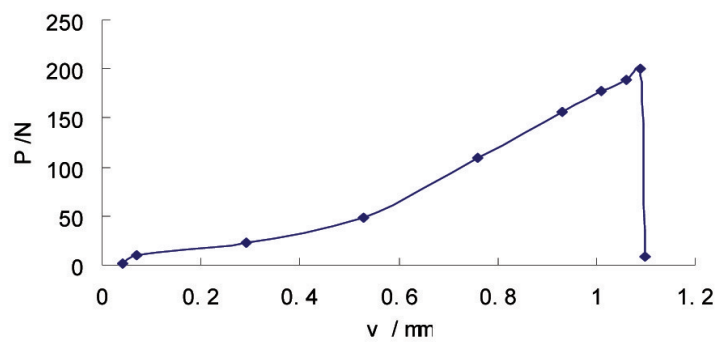

TR

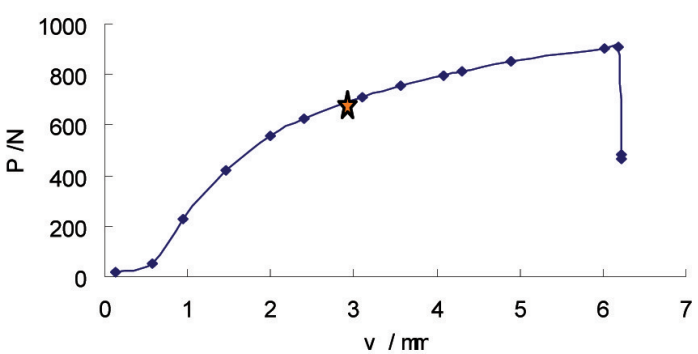

LT (Pinus bungeana)

FIGURE 4. Load and displacement curve for specimens during the second desorption stage al $20^{\circ} \mathrm{C}$ $(82.4 \% \mathrm{RH} \longrightarrow 35.2 \% \mathrm{RH})$

\section{Fracture toughness}

Seen from figure 5 , in the second desorption process $(82.4 \%$ to $35.2 \% \mathrm{RH})$, specimen fracture toughness decreased with elapsed desorption time. This was opposite to that observed in the first stage of desorption. Further examination for relationship between fracture toughness and moisture content is shown in Figure 6, below the fiber saturation point, for moisture content from 0 to $17 \%$, wood fracture toughness increased as MC increased, and reached the maximum value at the MC $17 \%$. The fracture toughness subsequently decreased with increasing of MC from $17 \%$ to $28 \%$. Similar results have been found for fracture load for resistance to cleavage could reach the maximum value at the moisture content $18 \%$ (Mamada and Aoki 1992). Futher work is needed to understand the reasons for such behavior.

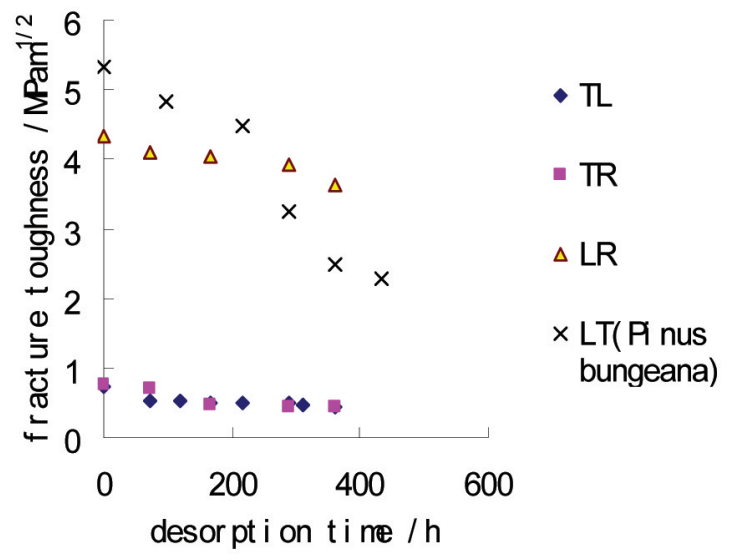

FIGURE 5 Fracture toughness of specimens during the second desorption stage at $20^{\circ} \mathrm{C}(82.4 \% \mathrm{RH} \longrightarrow 35.2 \% \mathrm{RH})$ 


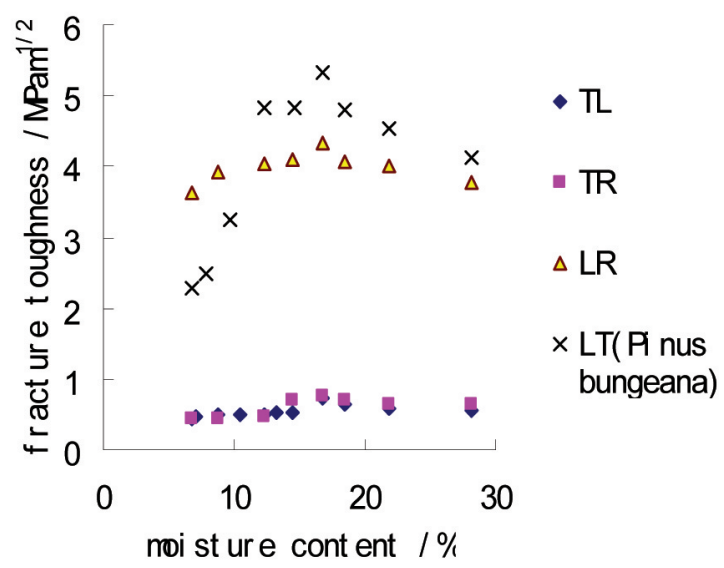

FIGURE 6 Relationship between moisture content and fracture toughness

\section{CONCLUSION}

In this work, it was found that for a given type of specimen, the crack propagation path in desorption follows a similar pattern to adsorption as observed in previous studies (Wang, et al., 2003).

Specimen crack orientation was found to influence crack propagation path. The direction of crack propagation for TL and TR specimens was basically along the initial crack direction, namely TL specimens propagate along the fiber direction, TR specimens along the radial direction or along wood ray direction.

Moisture content in the wood influenced wood fracture toughness at a given temperature. For wood moisture content change from fiber saturation point state to an equilibrium moisture content at $82 \% \mathrm{RH}$ and $20^{\circ} \mathrm{C}$, fracture toughness for all of the specimens gradually increased with elapsed time. However, with further desorption from $82 \% \mathrm{RH}$ to $35 \% \mathrm{RH}$, wood fracture toughness decreased with time.

\section{REFERENCES}

Chinese National Standard.1985. Standard test method for plane-strain fracture toughness of metallic materials. GB4161-84.

Chinese National Standard.1992. Method of sample tree collection for physical and mechanical tests of wood.GB 1927-91.

Kretschmann, D.E.; Green, D.W. 1996. Moisture content specific gravity for relationships for clear southern pine. In: Proceedings of the International Wood Engineering Conference.New Orleans, LA. pp. 536-542.

Smith, I.; Chui, Y.H. 1994. Factors affecting mode I fracture energy of plantation-grown red pine. Wood Sci. and Technol. 28:147-157.

Mamada, S.; Aoki, S. 1992. A test for wood dynamic cleavage. Bull.Niigata Univ. For. (25): 141146.

Wang, L. Y.; Lu, Z.Y.; Zhao, G.J. 2003. Wood fracture Pattern during the Water Adsorption Process. Holzforschung 57 (6): 639-643 